\title{
Mathematical Modeling of Cooperative E-Learning Performance in Face to Face Tutoring (Ant Colony System Approach)
}

\author{
doi:10.3991/ijac.v3i4.1471
}

\author{
Hassan. M. Mustafa \\ Al-Baha University, Al-Baha, Kingdom of Saudi Arabia.
}

\begin{abstract}
Investigational analy sis and evaluation of cooperative le arning phe nomenon is an inte rdisciplinary and challenging educational research issue. Educationalists have been inter esting in modeling of human's cooperative lea rning to investiga te its analog $y$ with some learning aspects of observed $s$ ocial in sect $b$ ehavior. $S$ pecifically, th is $p$ aper presents real istic mod eling in spired from interdisciplinary integrated fields of ecology, education , and a nimal behavior learning sciences. Presented modeling considers cooperative behavioral learning at ant co lony s ystem ( ACS). That's motivated by qualitative sim ulation results obtained after running of an ACS algor ithm searching for optimal solution of Travelling Salesman Proble $m$ (TSP). In the context of computational intellig ence; cooperativ e ACS algorithm reaches optima I TSP solution analogously to converg ence process of He bbian coincidence le arning paradigm. M oreover, suggested mathematical modeling presents diversity of positive in terdependence as pect ob served $d$ uring $h$ uman's interactive coo perative $I$ earning. I nterestingly, $p$ resented analysis and evaluation of mathematically modeled practical insights of a dopted phenomenon, may shed light on promising fu ture en hancement of cooperative lea rning $p$ erformance.
\end{abstract}

Index Terms-Ant Colony Sy stem Optimization; Cooperative Lea rning; Computational Intelligenc e; E-Learning Systems; Traveling Sales Man Problem.

\section{INTRODUCTION}

In face to face tutoring, the phase of interactive cooperative learning is an essential paradigm aiming to improve any Open Learning System (OLS) performance. It has been declared that cooperative learning among studying agents (fellows), contributes about one fourth of attained output learning achievement during face to face tutoring sessions at OLS [1]. Herein, the two notations of Open Learning and E-Learning are presented interchangeably.

Considering observed resemblance between human's interactive cooperative learning at OLS, and cooperative behavioral learning function (among ants) at Ant Colony System(ACS). Mathematical modeling for both disciplined learning paradigms has been presented commonly. In more details, this work has central concern with comparative analogy (resemblance) between algorithmic performance of cooperative learning among ants at (ACS) during searching for optimal solution of Travelling Salesman Problem (TSP) [2]. Versus human's cooperative learning performance observed among students at OLS during face to face tutoring sessions. Furthermore, in computational intelligence context; resemblance between two neuronal and non-neuronal disciplines observed in nature have been comparatively presented. They are respectively; cooperative behavioral learning among number of place field neuronal cells (at hippocampus rat's brain area) contributing in solving reconstruction problem inside 8-Figure maze[3]. Secondly, ants' collective behaviors using cooperative autocatalytic algorithm of ACS which performed for solving optimally TSP [2].

Referring to the neuronal discipline, the number of neuronal cells-contributing in reconstruction problem solution-performs enhancement for reaching convergence limit of learning error (Cramer-Rao bound)[4][5]. More precisely, presented rat's behavioral intelligence modeling illustrates the effect of increasing number of neurons upon enhanced (better) performance in reaching more accurate results during solving a pattern recognition (reconstruction)problem. Interestingly, number of neuronal cells has well resemblance to the number of contributing agents in natural cooperative learning phenomenon. That's observed in case of cooperation among either human learners (students), at e-learning system (in classrooms), or environmental agents (ants) of ACS during performing optimal solution of TSP .

Herein, cooperative learning performance is mathematically modeled in order to evaluate realistically cooperative tutoring sessions in an OLS. That's accomplished on the bases of optimal solution of TSP reached by an autocatalytic ACS algorithm. With dependence upon different communication levels among ACS agents (ants), optimal solutions of TSP obtained by various speeds. Analogously, consecutive interactive steps performed by fellow learners in OLS accumulate cooperative learning achievements in accordance with different intercommunication among learners. The introduced mathematical model represents quantitative mapping of qualitative results previously obtained after ACS performance evaluation according to either with or without intercommunication among ants [2][6].

Considering addressed metric time measurement (as a learning performance parameter) of learning convergence speed, it corresponds to learning response time. Practically, in fulfillment of some pre-assigned learning achievement (output level) at an educational system. It is observed to attain that achievement after some learning convergence period of time called as learning response time. The introduced mathematical modeling at this work is mainly supported by results of two recently published work. Firstly, that's deals with comparison between prin- 
ciples of biological information processing mechanisms in neural versus non-neural bio-systems [7].However, the other work presents evaluation of results obtained after a psycho-learning experiment carried on some animals versus optimal performance results of swarm intelligent (ACS) [8].

In more details, introduced modeling proved to perform realistically in resemblance to expected performance of mutual interactive cooperative learning. That's dependent upon diverse positive interdependence aspects among learning agents(either ants or students). Considering comparative context, analogy between two types of distinct cooperative learning performance associated with computational intelligence agents (ants \& neurons) has been verified by obtained interesting results. More precisely, obtained comparative results demonstrated the increasing number effect of cooperative learning agents (either neural cells or ants) on learning performance at both types. One particular virtue of swarm intelligence analogy with cooperative learning phenomenon is that it provides a means of e-learning performance enhancement in accordance with positive interdependence communication aspects among learning agents.

Conclusively, presented comparative mathematical modeling as well as results obtained after programs' running, may be promising in optimal improvement of educational achievement(s) at face to face tutoring sessions during Open Learning Processes.

It is Interesting to announce that some challenging educational issues have been previously studied and investigated systematically. Noting those investigational studies were inspired by natural observations of animals' behavioural learning. After analysis and evaluation of suggested issues, valuable results have been obtained and recently published. Examples of such investigated issues are: How Should Reading Be Taught? [9-12], and investigational analysis of learning creativity[13][14]. It is worthy to note that obtained results are inspired by natural observations of other animals' behavioural intelligence (dogs, cats, and rats) rather than ants' behaviour observed in ACS.

The rest of this paper organized as follows. At the next second section, an overview revising autocatalytic behavioural learning process among agents ACS (ants), is presented. That's in correspondence with positive interdependence feed back during transportation process of food. The quantitative mapping of qualitative results previously obtained after ACS performance evaluation is introduced at the third section. At the fourth section, detailed analysis and evaluation of Hebbian coincidence learning performance is presented as unsupervised paradigm based on associated synaptic connectivity patterns. The generalized mathematical modeling of ACS performance is formulated with resemblance to cooperative learning performance at the fifth section. Finally, at the last sixth section, some conclusive remarks and discussions are given. By the end of this work, an Appendix is attached which illustrates a simplified macro level flowchart which describes algorithmic steps of simulation program for Hebbian learning using Artificial Neural Network ANN.

\section{REVISING ANT COLONy System PERFoRMANCE}

The field of swarm intelligence deals with systems composed of many individuals (agents). That coordinate cooperative behavioral learning among ants using decen- tralized control and self-organization. In this section, swarm intelligence of ACS considered as observed by many cooperative individuals (ants)in natural environment. These ants observed to perform storing food function of by repetitive movements on a straight line connecting a food source to their nest [6]. Referring to Figure.1 shown in below, a pheromone trail is the primary means for ants to maintain bidirectional walking (back and fro) in straight line. Ants deposit a certain amount of pheromone while walking, and each ant probabilistically prefers to follow a direction rich in pheromone. Referring to Fig. $1 \mathrm{~B}$, this elementary behaviour of real ants can be used to explain how they can find the shortest path that reconnects a broken line after the sudden appearance of an unexpected obstacle has interrupted the initial path. In fact, once the obstacle has appeared, those ants which are just in front of the obstacle cannot continue to follow the pheromone trail and therefore they have to choose between turning right or left. In this situation we can expect half the ants to choose to turn right and the other half to turn left. A very similar situation can be found on the other side of the obstacle (Figure 1C). It is interesting to note that those ants which choose, by chance, the shorter path around the obstacle will more rapidly reconstitute the interrupted pheromone trail compared to those which choose the longer path. Thus, the shorter path will receive a greater amount of pheromone per time unit and in turn a larger number of ants will choose the shorter path. Due to this positive feedback (autocatalytic) process, all the ants will rapidly choose the shorter path (Figure 1D).

The most interesting aspect of introduced autocatalytic process is that finding the shortest path around the obstacle seems to be an emergent property of the interaction between the obstacle shape and ants distributed behaviour: Although all ants move at approximately the same speed and deposit a pheromone trail at approximately the same rate, it is a fact that it takes longer to contour obstacles on their longer side than on their shorter side which makes the pheromone trail accumulate quicker on the shorter side. It is the ants' preference for higher pheromone trail levels which makes this accumulation still quicker on the shorter path. This process is adapted with the existence of an obstacle through the pathway from nest to source and vice versa, however, more detailed illustrations are given at [6].Therein, ACS performance obeys computational biology algorithm used to solve travelling salesman problem TSP optimally.

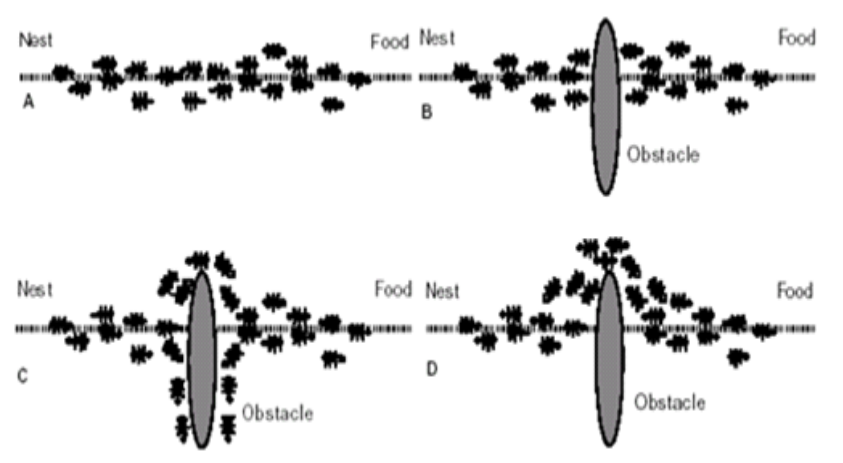

Figure 1. Illustrates the transportation process of food back and fro, (from food source) to food store (nest). Detailed illustrations are given through the text at [6] 


\section{A. Qualitative Analogy Between ACS Autocatalytic} Process And Cooperative Learning Performance

At this subsection; qualitative illustration of obtained optimal solution of TSP considering intercommunication among ants, is shown graphically at Figure 2. This solution mainly depends upon the most interesting aspect of ACS autocatalytic process. That's finding the shortest path around the obstacle seems to be an emergent property of the interaction between the obstacle shape and ants distributed feedback behaviour. Although all ants move at approximately the same speed and deposit a pheromone trail at approximately the same rate, it is a fact that it takes longer to contour obstacles on their longer side than on their shorter side which makes the pheromone trail accumulate quicker on the shorter side. It is the ant's preference for higher pheromone trail levels which makes this accumulation still quicker on the shorter path. Referring to cooperation learning paradigms, presented ACS autocatalytic process is similarly a form of collaboration where ants are working together to accomplish shared goals[15]. Moreover, it is characterized by performing positive intercommunication aspect similar to the most important element in structuring cooperative human learning, that's positive interdependence feedback [16] .Consequently, presented cooperative behavioural animal learning seems to have well resemblance with unsupervised Hebbian learning paradigm [17-20].

\section{B. Intelligent Technique of Leptothorax albipennis ACS Versus Cooperative Learning Performance}

Conversely to above presented computational biology algorithm which applied for solving TSP ; by referring to two recently published research work. An overview on decision-making in a decentralized(distributed) biological system represented by ACS, is presented at [21][22].Therein, a specific type of ant colony systems called Temnothorax albipennis performs a paradigmatic decentralized decision-making based on learning/teaching technique known as tandem running. its individual agents (ants) adopt tandem running which is an intelligent behavioral teaching technique[22]. Colonies of that ant type have been shown flexibly to compromise accuracy for speed. Briefly, in case of one ant running to lead another ant moving from the nest to food, both leader and follower are analogous to teacher and pupil. Both are acutely sensitively adaptive to progressing of their partner. This learning technique involves teaching by interactive feedback between two ants controlling trade-off between speed and accuracy[22].

Furthermore, in OLS, this supervised learning paradigm contributes another one forth of attained learning achievement(output)during face to face tutoring sessions ; as introduced at [1]. Referring to Figure 3, it illustrates that learning paradigm of tandem running technique involves bidirectional feedback between teacher and pupil corresponding to leader and follower respectively. Furthermore at this figure, depicted block named as (Follower/Leader) suggests that tandem followers after learning their lessons so well, that they often become tandem leaders.

In cooperative learning context, the above type of ACS behavioral learning performance [22][23] is analogous to what could be observed in classrooms; if one agent (student) behaves independently upon other agents' achievements [15]. So, it described as teacher-centered providing

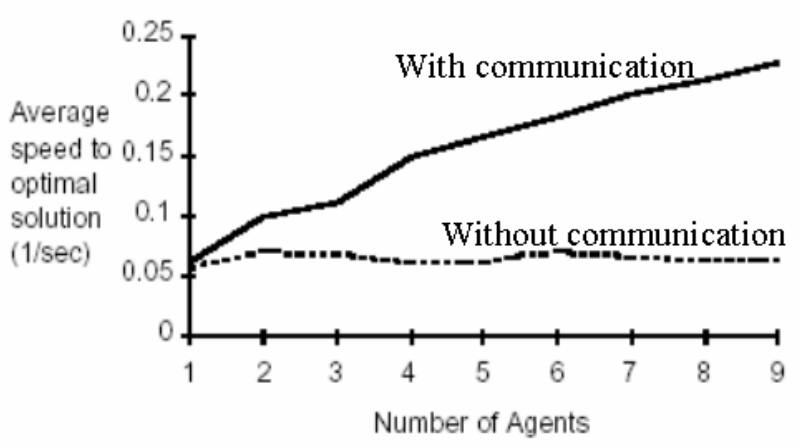

Figure 2. Illustrates qualitative effect of intercommunication among ACS agents (ants) depending on autocatalytic learning performance to solve optimally TSP adapted from [6].

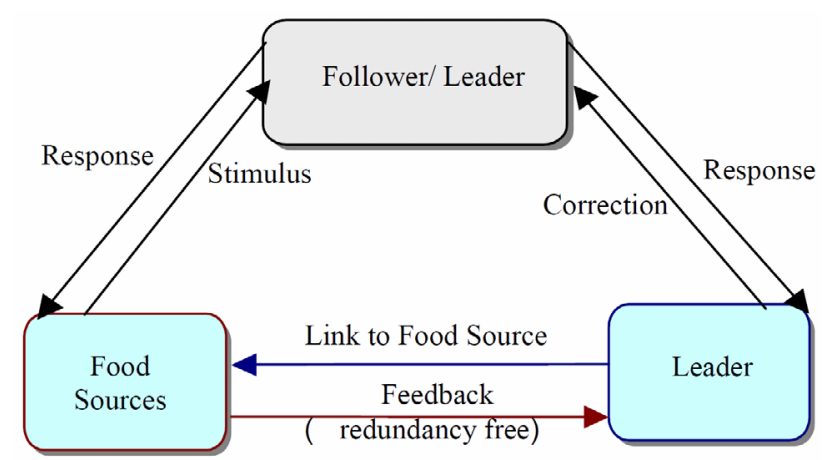

Figure 3. Illustrates a schematic diagram for tandem running technique involves bidirectional feedback.

individual learning which implies that leader ant (teacher) can transfer knowledge and cognitive skill to the learner (another ant) [15][16] . Accordingly, via That teachercentered type of learning the teacher provides the major source of information, and feedback [24]. Conclusively, ANN models based on supervised learning paradigm are relevant for realistic simulation of cooperative teachercentered learning performance [15][16].

\section{QUANTITATIVE PERFORMANCE OF ACS}

\section{A. Representation of ACS Performance Using Set of Odd Sigmoid Function Curves}

At this piece of research, comparative analogy between simulation results obtained after Hebbian learning modelling, and that concerned with ACS performance during solving TSP optimally; has been with central concern as presented at next sections.

This subsection presents simulation results after Hebbian learning modeling to illustrate quantitatively ACS performance. Obviously, number of intelligent agents contributing to solution of TSP by ACS correspondent well to neurons' number contributing in Hebbian learning models.

Referring to figure 2 (adapted from [6]), it illustrates qualitatively performance differences for reaching optimal TSP solutions. Those differences presented in accordance with different synergistic communication levels among ACS agents (ants). Consequently, different average output speeds are developed following changes of different intercommunication levels to reach TSP optimum solution. These changes are quantitatively analogous to different gain factor values $\lambda$ given by odd sigmoid function shown by the equation: 


$$
f(n)=\alpha\left(\frac{1-e^{-\lambda n}}{1+e^{-\lambda n}}\right)(1)
$$

Where $\alpha$ is an amplification factor representing asymptotic value for maximum average speed to reach optimized solutions of TSP,

n.....denotes the number of ants contributing in solving TSP, and $\lambda \ldots \ldots \ldots$ is the gain factor (slope) denoting changes according to different intercommunication levels among ACS agents. Moreover referring to figure 2, by relevant choice for values of $f(n)$ function's parameters $(\lambda$ $\& \alpha)$ such that $(f(1)=0.06)$ and

$(f(\infty)=\alpha$ ) (noting the average speed converges to $\alpha$ measured in $\mathbf{H Z})$.

Consequently, the value of $\lambda$ actually is directly proportional to pheromone trail mediated communication among agents(ants) of ACS. The performance of the synergistic effect of ACS referred to the generalized sigmoid function of discrete (n) integer Positive values. (representing number of ants). Moreover, the two extreme limits values of $f$ (n) are as follows. It equals to minimum zero value as the number of ants vanishes $(n=0)$. Nevertheless, the function reaches the optimal speed reaches at value of $\alpha$ as the maximum number of ants tents to infinity $(n=\infty)$. in other words, this function declares that gain factor value of odd sigmoid function is a direct quantitative measure for intercommunications level among ants in ACS . I.e. the value of gain factor $(\lambda)$ proportions is directly to pheromone trail mediated communication among ACS agents (ants) performing cooperative learning. Accordingly, assuming no intercommunications among ACS agents ; global learning performance of ACS has become nearly parallel to $X$-axis corresponding to slope, $\lambda=0$. In this case, increasing number of ants presented on $X$-axis results in impossibility to reach optimal solution. Equivalently, optimal solution is reached (virtually) by infinite number of ants contributing in ACS behavioral cooperative learning. Interestingly, the above obtained results for solving TSP are analogous to Hebbian learning simulation performance given by set of graphs shown at Figure 4. That's illustrating quantitatively the correspondent analogy between intercommunication levels and different gain factor values at fixed learning rate value and number of training cycles. The tabulated computer simulation results given at Table I, are presented after considering learning rate $(\eta)=0.1$, and gain factor $(\lambda)=0.5$. Interestingly, these results representing response time and speed for convergence are corresponding well to ACS performance of the qualitative (with communication) level given at Figure 2. At Appendix I, a simplified macro level flowchart is shown describing algorithmic steps for simulation program of Hebbian learning using Artificial Neural Networks. After running that program, three achievement curves corresponding to three , different gain factor values are obtained (as shown at Figure 4). Furthermore, a generalized set of curves presenting normalized achievements for different gain factor values (versus number of training cycles), is shown at Figure 6 , in the fifth section.

\section{B. Rat's Behavioral Learning Model While Solving Reconstruction Problem Inside 8-Figure Maze}

Referring to psycho-experimental work concerned with behavioral learning of a mouse inside 8-Figure maze, results obtained while solving the reconstruction problem presented in two (graphical and tabulated) forms. Graphi-

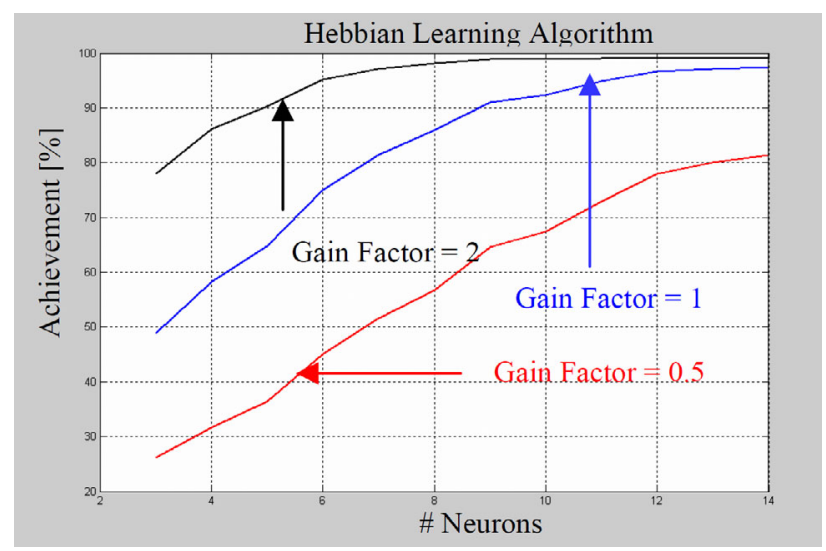

Figure 4. Illustrate learning performance to get accurate solution with different gain factors $0.05,1$, and 2 , while \#cycles $=300$ and Learning rate $=0.3$

TABLE I.

ILLUSTRATES THE RELATION BETWEEN INCREASING NEURONS ' NUMBER VESUS RESPONSE TIME AND AVARAGE LEARNING SPEED CONSIDERING HEBBIAN LEARNING RULE.

\begin{tabular}{|c|r|c|c|c|c|c|}
\hline $\begin{array}{c}\text { Number of } \\
\text { Neurons }\end{array}$ & 35 & & 7 & 9 & 11 & 14 \\
\hline $\begin{array}{c}\text { Response } \\
\text { Time } \\
\text { (\# Cycles) }\end{array}$ & 79.761. & 8 & 36.9 & 19.8 & 14.8 & 7.3 \\
\hline $\begin{array}{c}\text { Average } \\
\text { Learning } \\
\text { Speed (1/Cyc.) }\end{array}$ & 0.0130. & 016 & 0.027 & 0.051 & 0.068 & 0.137 \\
\hline
\end{tabular}

cal results are shown by convergence curve at Figure 5, however that tabulated are given at Table II. That experimentally measured results are based on pulsed neuron spikes at hippocampus of the mouse brain. According to obtained data, the error value seems to decrease similarly to an exponential curve decays to some limit value versus the place field cells.

Modeling of the suggested reconstruction problem verification on the basis of Bayesian reconstruction to estimate the position of the rat in 8-figure maze(more details are introduced at the fifth section). Noting the value of mean error converges (by increase of number of cells) to some limit, excluded as Cramer-Rao bound. That limiting bound is based on Fisher's information given as tabulated results in the above and derived from [4].That implies LMS algorithm is valid and obeys the curve shown at in blow.

The value of mean error converges (i.e. by the increase of number of cells) to some limit, excluded as CramerRao bound. The limit bound is based on Fisher's information given in Table II, that's derived from [4]. This implies the curve as shown in Figure 5 obeys the validity of Least Mean Square (LMS) algorithm [8][11].

\section{REVISING OF HEBBIAN COINCIDENCE LEARNING PERFORMANCE}

Modeling of Hebbian learning rule performance is classified as unsupervised coincidence learning paradigm based on performing of detection process concerned with any environmental pattern. That's by using associated synaptic connectivity(weight) pattern to the developed input environmental pattern [14][25-27]. 


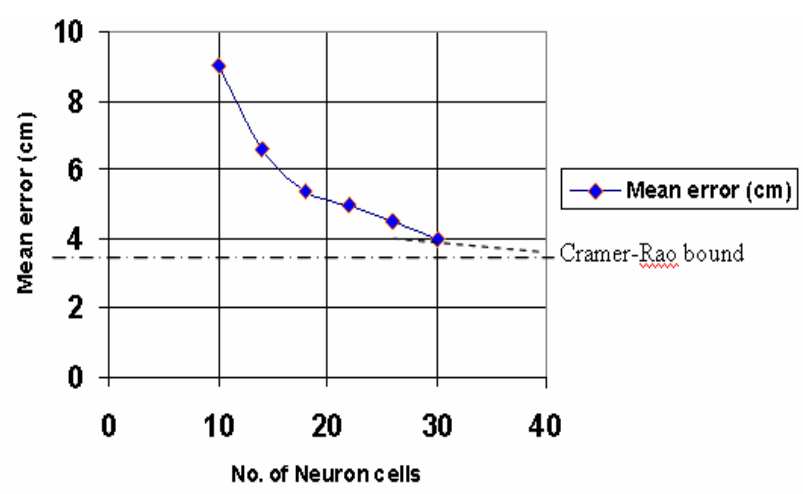

Figure 5. The dashed line indicates the approach to Cramer-Rao bound based on Fisher information [4].

TABLE II.

RELATION BETWEEN NUMBER OF CELLS AND MEAN ERROR IN SOLVING RECONSTRUCTION PROBLEM

\begin{tabular}{|r|c|c|c|c|c|c|}
\hline No. of neuron cells & 14 & 18 & 22 & 26 & 30 & \\
\hline Mean error (cm) & 9 & 5.6 & 4 & 4 & 5 & 4 \\
\hline
\end{tabular}

Referring to above two figures (2\&4), convergence of two cooperative learning processes is dependent upon capability of contributing cooperative agents (either ants in ACS or neurons in human brain ) in reaching desired solution of suggested problem. Accordingly, it seems very relevant to adopt realistic modeling of Hebbian coincidence detection using ANN to simulate performance resemblance between ACS, and that for human brain learning function. More precisely, that resemblance introduced by using behavioral learning modeling of neuronal synaptic plasticity (dynamical weights connectivity). So, biological neuronal patterns inside brain are well recognized and evaluated via coincidence detection learning of some environmental pattern on the basis of Hebbian learning rule. In some details, by referring to [25][26], learning coincidence detection is presented as follows. The angle $\alpha$ between training weight vector and the input vector to be detected. Consequently for ideal detection learning tan $(\alpha)$ equals zero. However, on the extreme value impossibility of learning detection occurs when $\alpha$ equals $\Pi / 2$. So, under the assumption that $\lambda=\frac{1}{\tan \alpha}$ the value of $\lambda$ ranges from zero to infinity.

Furthermore, by referring to [27], the results of learning process considering Hebbian rule are shown by the equation:

$$
y=\left(1-e^{-\lambda t}\right)
$$

In other words, this value of $\lambda$ corresponds to the gain factor (slope) in classical sigmoid function. This equation performs analogously, in agreement with gain factor (slope) presented by classical sigmoid function given by the equation :

$$
y(t)=\frac{1}{1+e^{-\lambda t}}
$$

The exponentially increasing function (1) behaves in a similar way as the sigmoid function as it saturated at unity value $(y(t)=1)$ when learning / training time approaches to infinity. More precisely, equation (1) performs closely similar to odd sigmoid function given as:

$$
y(t)=\frac{1-e^{-\lambda t}}{1+e^{-\lambda t}} \quad \text { (4) for } 0 \leq t \leq \infty
$$

Finally, referring to Figure 5, it represents different three individual levels of learning performance curves. Firstly, curve Y2 is an equalized representation of both forgetting and learning factors [25][27]. So, it simulates group of learners with normalized (natural) performance. However, curve Y1 simulates lower (inferior level) learning performance, i.e. individuals characterized by learning disability. Conversely, the curve Y3 indicates better learning performance than normalized level (of synaptic connectivity), simulated by learning performance curve Y2.,i.e. it simulates individuals having intrinsic learning creativity. The three presented graphs are having good resemblance to different three classified ant colonies that having three different levels of intercommunication among ants. That case is analogous to cooperative learning phenomenon with different positive interdependence aspects among students.

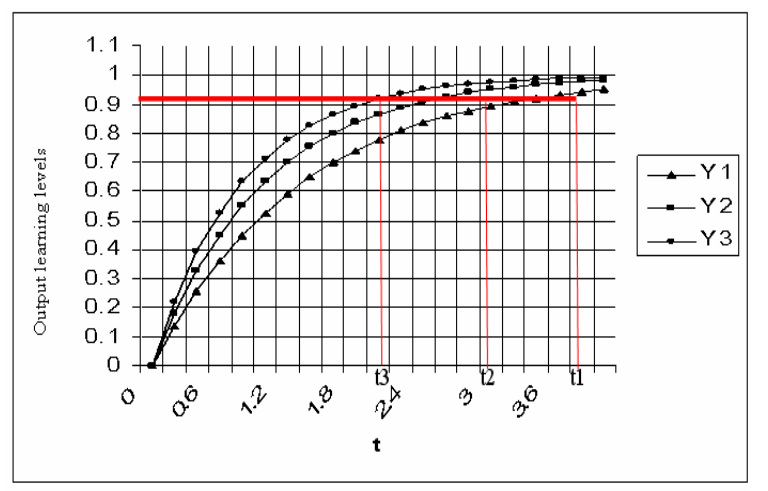

Figure 5. Illustrates three different learning performance curves $\mathrm{Y}_{1}, \mathrm{Y}_{2}$ and $\mathrm{Y}_{3}$ that converge at time $\mathrm{t}_{1}, \mathrm{t}_{2}$ and $\mathrm{t}_{3}$ considering different $\lambda$ (gain factor values) presented as slope $\left(\lambda=\frac{1}{\tan \alpha}\right)$.

\section{MATHEMATICAL FORMULATION OF ACS PERFORMANCE}

\section{A. Rat's Reconstruction Problem Versus ACS}

Autocatalytic Learning Process For Solving TSP.

Referring to [4] the timing of spikes in a population of neurons_can be used to reconstruct a physical variable is the reconstruction of the location of a rat in its environment from the place fields of neurons in the hippocampus of the rat. In the experiment reported here, the firing partterns of 25 cells were simultaneously recorded from a freely moving rat, [3]. The place cells were silent most of the time, and they fired maximally only when the animal's head was within restricted region in the environment called its place field [5]. The reconstruction problem was to determine the rat's position based on the spike firing times of the place cells.

Bayesian reconstruction was used to estimate the position of the rat in the figure- 8 maze shown in above figure 2,that according to [4]. Assume that a population of $\mathrm{N}$ neurons encodes several variables $\left(x_{1}, x_{2} \ldots \ldots\right)$, which will be written as vector $x$. From the number of spikes $\mathrm{n}=\left(n_{1}, n_{2}, \ldots \ldots n_{\mathrm{N}}\right)$ fired by the $N$ neurons within a time interval $\tau$, we want to estimate the value of $x$ using the Bayes rule for conditional probability: 


\section{Mathematical Modeling of CoOPerative E-Learning Performance in Face to Face Tutoring (ANT COLONY SYSTEM APPROACH)}

$P(\mathrm{x} \mid \mathrm{n})=P(\mathrm{n} \mid \mathrm{x}) P(\mathrm{x}) / P(\mathrm{n})$

Assuming independent Poisson spike statistics. The final formula reads

$$
P(x \mid n)=k P(x)\left(\prod_{i=1}^{N} f i(x)^{n i}\right) \exp \left(-\tau \sum_{i=1}^{N} f i(x)\right)
$$

Where $k$ is a normalization constant, $P(\mathrm{x})$ is the prior probability, and $f_{\mathrm{i}}(\mathrm{x})$ is the measured tuning function, i.e. the average firing rate of neuron $i$ for each variable value $\mathrm{x}$. The most probable value of $\mathrm{x}$ can thus be obtained by finding the $\mathrm{x}$ that maximizes $P(\mathrm{x} \mid \mathrm{n})$, namely,

$$
\hat{x}=\arg \max P(x \mid n)
$$

By sliding the time window forward, the entire time course of $x$ can be reconstructed from the time varyingactivity of the neural population.

The above equation for solving reconstruction problem (corresponding to the most probable value of $\mathrm{x}$ ) seems to be very similar to the equation searching for optimum solution considering TSP reached by ACS (for random variable $\mathrm{S}$ ) as follows.

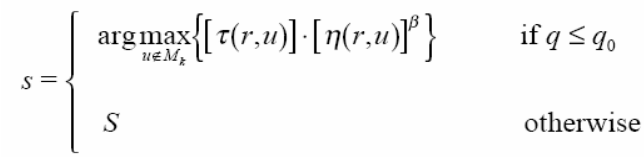

where $\tau(\mathrm{r}, \mathrm{u})$ is the amount of pheromone trail on edge $(\mathrm{r}, \mathrm{u}), \eta(\mathrm{r}, \mathrm{u})$ is a heuristic function, which was chosen to be the inverse of the distance between cities $r$ and $u, \beta$ is a parameter which weighs the relative importance of pheromone trail and of closeness, $\mathrm{q}$ is value chosen randomly with uniform probability in $[0,1], \mathrm{q}_{0}\left(0 \leq \mathrm{q}_{0} \leq 1\right)$ is a parameter, $M_{k}$ is memory storage for $\mathrm{k}$ ants activities, and $\mathrm{S}$ is a random variable selected according to some probability distribution [6][28].

\section{B. B. Generalized Model Of Learning Performance \\ Curves For Humans' Cooperative Learning And ACS}

Normalized behavioral model of ACS (Optimization Algorithm), adopted for solving TSP[6]. It considers the changes of communication levels (indicated by $\lambda$ parameter). This parameter value causes changing of the speeds for reaching optimum solutions following the equation:.

$$
y(n)=(1-\exp (-\lambda i(n-1))) /(1+\exp (-\lambda i(n-1)))
$$

where $\lambda \mathrm{i}$ represents one of gain factors (slopes) for sigmoid function.

Conversely to above set of sigmoid functions, a normalized set of decayed exponential curves are given in below at Figure 7 . By following formula where suggested $\left(\eta_{\mathrm{i}}\right)$ as learning rate factor. These learning rate factors are denoted by $\left(\eta_{\mathrm{i}}\right)$. These factors are mathematically presented after normalization by different learning performance curves (at Figure 7) as follows:

$$
y(n)=\exp \left(-\eta_{i}(n-1)\right)
$$

where (n) is the number of training cycles.

Referring to Figures (8\&9) given in below, the effect of gain factor as well as learning rate changes on cooperative learning performance is introduced grapthically .It is very interesting to note that analysis of introduced effect is supported well by the decayed graphs described by equation (10) and illustrated set of curves at Figure 7.

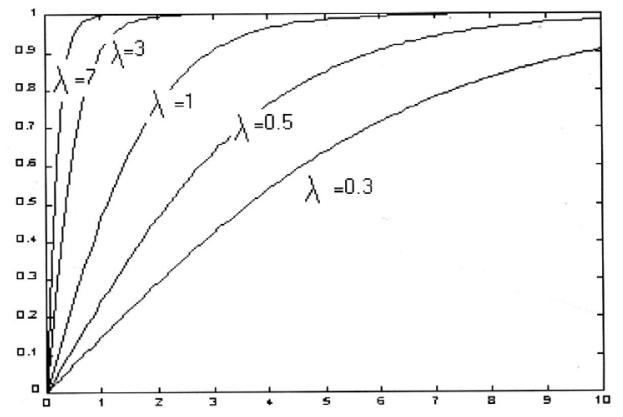

Figure 6. Graphical representation of learning performance of model with different gain factor values $(\lambda)$ adapted from, $[8]$.

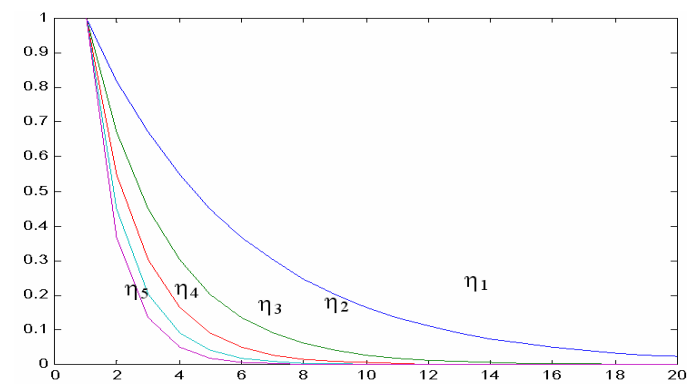

Figure 7. Illustrates different learning performance curves fordifferent learning rate values $(\eta)$.

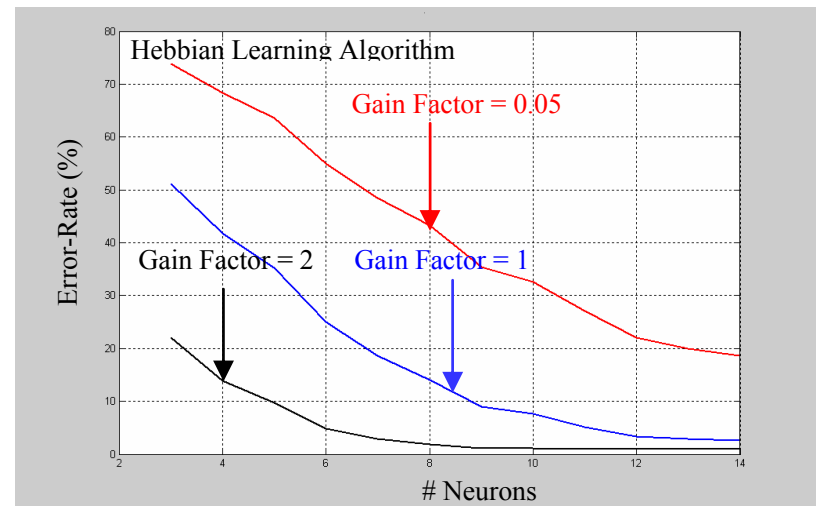

Figure 8. Illustrate Hebbian learning performance error-rate with different gain factors : $0.05,1$, and 2, while \#cycles = 300 and Learning rate $=0.3$

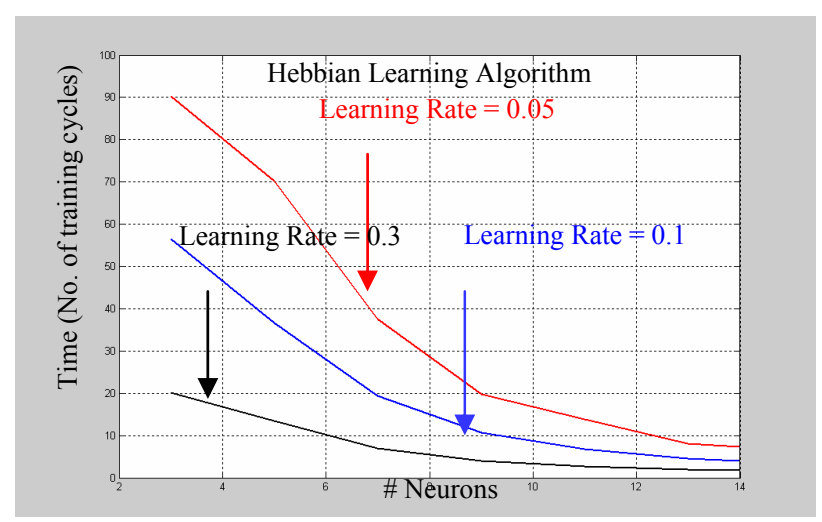

Figure 9. Illustrates Hebbian learning performance \& time factor with considering three different learning rates: $0.05,0.1$, and 0.3 for gain factor $=0.5$ 


\section{Mathematical Modeling of CoOPerative E-Learning Performance in Face to Face Tutoring (ANT COLONY SYSTEM APPROACH)}

\section{CONCLUSIONS AND DSCUSSIONS}

In nature, response time needed to perform some desired goal by animal brain dependent upon stored experience inside brain. So, execution time is a very effective factor to evaluate performance of simulation simulated educational phenomena. This factor is very relevant to evaluate computational power for any computerized modelled system [29]. Obviously, such computational power analogously corresponds to intelligence needed to solve some complex modelled problem(s).

Co-operative learning paradigm adopted in e-learning systems aiming to improve output learning achievements. That's positively correlated with the cooperating students who work collaboratively in good level of intercommunication among students towards accomplish of a common goal [15]. Usually, students suggested to work together in small clusters or groups with good level of communication among them. This approach is in good resemblance to ants' behavior at ACS where effectiveness of co-operative learning promotes mainly positive interdependence .

The following three interesting conclusive remarks are valuable:

1. The number of trials during Hebbian Coencidence learning are in good resemblance with number of agents (ants) and the number of neurons as stored experience due to interaction with environment [30]. (see Figure 2, and Figure 4).

2. The existence of an obstacle at some point of ants' pathway is analogous to noisy applied when training some neural model. In accordance to the asymmetry of obstacles' shape, the time needed to find the shorter pathway (Solution of TSP)is directly proportional [8][31].

3. The stored experience during Hebbian process and computational intelligence of ACS are both analogues to the needed CPU time in order to develop minimum error for reaching optimum learning output (solution).

Finally, presented analysis and evaluation herein -based on suggested mathematical modelling- may shed light on promising enhancement of cooperative learning performance. That's by considering obtained interesting resemblance of cooperative learning phenomenon with unsupervised Hebbian coincidence learning rule.

\section{REFERENCES}

[1] H. Al-Ajroush, " Associate Manual for Learners' Support in Face to Face Tuition" Arab Open University (KSA),May 2004.

[2] Eric Bonabeau, and Guy Theraulaz, "Swarm Smarts" Majallat Aloloom, May 2001, vol 17, No 5, pp 4-12.

[3] Sejnowski, T.J: Neural pulse coding" foreword article for (Pulsed neural networks), MIT press, 1999, pp 13-23,1999.

[4] Zhang et al. Interpreting neuronal population activity by reconstruction." Journal of Neurophysiology, 79:1017-44, 1998.

[5] Wilson, M. A. and McNaughton, B. L., 1993: Dynamics of the hippocampal ensemble code for space. Science, 261: 1055-8. doi:10.1126/science. 8351520

[6] Dorigo, M. et al., Ant colonies for the traveling salesman problem, A set of papers at a web site http://www.iridia.ulb.ac.be/ dorigo/aco/aco.html ,1997.

[7] H.M. Hassan "On Principles of Biological Information Processing Concerned with Learning Convergence Mechanism in Neural and Non-Neural Bio-Systems." Published at IEEE conference, CIMCA 2005 , 28-30 Nov.2005, Vienna, Austria.
[8] H. M. Hassan "On Learning Performance Evaluation for Some Psycho-Learning Experimental Work versus an Optimal Swarm Intelligent System." Published at IEEE Symposium on Signal Processing and Information Technology ISSPIT, on 18-20 Dec.2005.Athens, Greece.

[9] keith Rayner, BarbaraR. Foorman, Charles A. Perfetti, David Pesetsky, Mark S. Seiedenberg: How Should Reading Be Taught? Majallat Aloloom, 2003, vol.19, number 6/7 pp.4-11.

[10] H.M. Hassan "On Mathematical Analysis, and Evaluation of Phonics Method for Teaching of Reading Using Artificial Neural. Network Models" published at SIMMOD 2005, pp 254-262.

[11] H.M.Hassan, Ayoub Al-Hamadi, and Saleh Al-Saleem "Towards Evaluation of Phonics Method for Teaching of Reading Using Artificial Neural Networks (A Cognitive Modelling Approach)", IEEE Symposium on Signal Processing and Information Technology ISSPIT 2007 , Cairo-Egypt.

[12] H.M.Hassan, S. Al-Saleem "On Artificial Neural Network Application for Modeling of Teaching Reading Using Phonics Methodology (Mathematical Approach)", published at the 6th International Conference on Electrical Engineering, ICEENG 2008, M.T.C. on 25-27 May, Cairo, Egypt.

[13] H.M.Hassan," A Comparative Analogy of Quantified Learning Creativity in Humans Versus Behavioral Learning Performance in Animals: Cats, Dogs, Ants, and Rats.(A Conceptual Overview), published at WSSEC08 conference held on 18-22 August 2008, Derry, Northern Ireland .

[14] H.M.Hassan, "On Analysis of Quantifying Learning Creativity Phenomenon Considering Brain Synaptic Plasticity", published at WSSEC08 conference held on 18-22 August 2008, Derry, Northern Ireland.

[15] Johnson D, Johnson R. (1991) Learning Together and Alone ed3.;Allyn \& Bacon, Sydney, pp.2.

[16] PLSB, Professional Learning Services Branch, 1999, Five Basic Elements of Cooperative Learning [Online access 14/6/02] URL: http://web.ec.tased.edu.au/las/sose/fiveelem.htm

[17] Hebb, D.O, 1949.: The organization of Behavior, A Neuropsychological Theory, New York, Wiley.

[18] Hampson, S.E. Connectionist Problem Solving, Computational Aspects of Biological Learning, Berlin, Birkhouser, 1990.

[19] Haykin S., " Neural Networks, Englewood Cliffs, NJ: PrenticeHall, pp. 50-60, 1999.

[20] Hassan H. and Watany M., "on comparative evaluation and analogy for Pavlovian and Throndikian psycho-learning experimental processes using bioinformatics modeling", AUEJ, 6,3, 424-432, july, 2003.

[21] Rechardson T.,Franks N.R. "Teaching in tandem-running ants", Nature, Jan 2006,12;439(7073):153.

[22] Marshall JA, Dornhaus A, Franks NR, Kovacs T."Noise, cost and speed-accuracy trade-offs: decision-making in a decentralized system" J R Soc Interface. Royal Society Publishing ,2006 Apr 22;3(7):243-54.

[23] Simon Garnier, Jacques Gautrais, and Guy Theraulaz" The biological principles of swarm intelligence "Swarm Intelligence Journal Volume 1, Number 1 / June, 2007 pp.3-31

[24] Salomon, g. Perkins, D. Theroux, P, 2001 Comparing Traditional Teaching and Student Centered, Collaborative Learning Online, accessed 14/6/02]URL: http://shaw.ca/priscillatheroux/collabora tive.html

[25] Tsien, Joe. Z., Enhancing the link between Hebb's coincidence detection and memory formation" in current opinion in Neurobiology, vol. 10, No. 2, (2000).

[26] Joe Z. Tsien 2001“ Building a brainier Mouse" Scientific American, Majallat Aloloom, vol. 17, No. 5, pp. 28-35.

[27] H. M. Hassan "On Quantitative Mathematical Evaluation of Long Term Potentiation and Depression Phenomena Using Neural Network Modeling" published at SIMMOD 2005, 17-19 Jan.2005, pp 237-241.

[28] Marco Dorigo , and Luca Maria Gambardella "Ant Colony System:A Cooperative Learning Approach to the Traveling Salesman Problem" published at the IEEE Transactions on Evolutionary Computation, Vol.1, No.1, 1997.

[29] Mariarty,E.D et al,1999: Evolvolutionary algorithms for reinforcement learning, journal of AI research 11:241-276. 
[30] Fukaya, M., et al. Two level Neural Networks: Learning by Interaction with Environment, 1st ICNN, San Diego,1988.

[31] H.M.Hassan, Ayoub Al-Hamadi, "On Comparative Evaluation of Thorndike's Psycho-Learning Experimental Work Versus an Optimal Swarm Intelligent System", published at IEEE conference, CIMCA 2008 Vienna, Austria (10-12 Dec. 2008).

\section{AUTHOR}

H. M. Mustafa is with Computer Engineering Department, Faculty of Engineering, On leave from Banha Uni- versity Egypt, Al-Baha, Kingdom of Saudi Arabia. (Email: mustafa_hasan47@yahoo.com)

Submitted October $10^{\text {th }}, 2010$. Published as resubmitted by the authors October $17^{\text {th }}, 2010$.

\section{APPENDIX}

The shown simplified macro-level flowchart in below briefly describes algorithmic steps for realistic simulation learning program using Artificial Neural Networks. Obtained results after running of that program, are shown at three figures $(4,8$, and 9$)$ at the paper text.

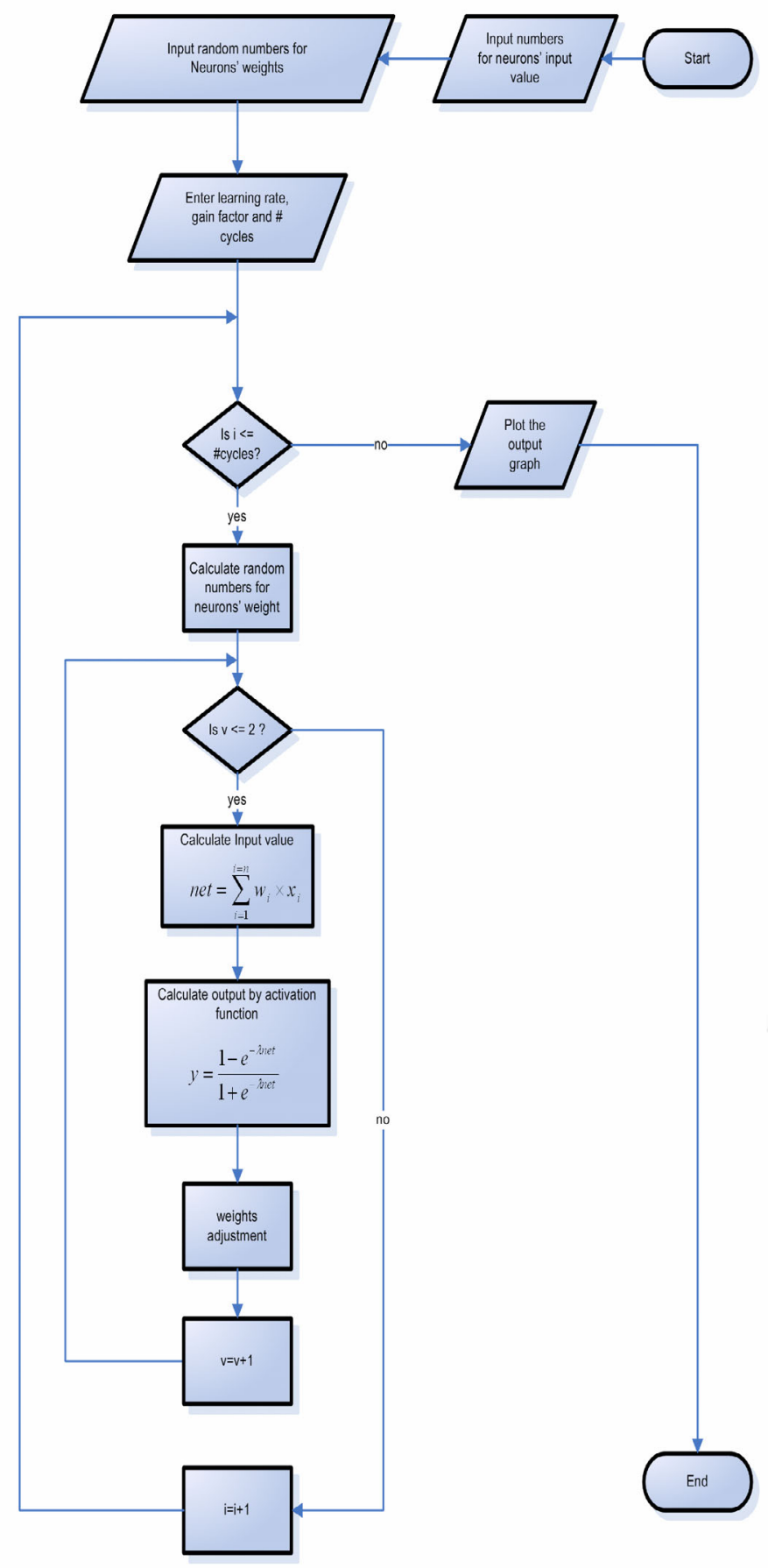

